\title{
Some Connections between Heyting Valued Set Theory and Algebraic Geometry
}

\author{
-Prolegomena to Intuitionistic Algebraic Geometry-
}

\author{
By
}

\author{
Hirokazu NisHimuRA*
}

\begin{abstract}
Rousseau [10] has shown that classical or standard function theory of $n$ variables is no other than intuitionistic function theory of one variable over $\mathbb{C}^{n-1}$. Similar works have been done by Nishimura [9] in the realm of Sato hyperfunctions and by Takeuti and Titani [16] in the realm of complex manifolds. The main purpose of this paper is to pursue similar results in the arena of algebraic geometry. Since we would like to do so in an intuitionistically valid manner, we reconstruct some rudiments of algebraic geometry, using the complete Heyting algebra of radical ideals in place of the space of prime ideals with Zariski topology as the starting point of our scheme theory.
\end{abstract}

\section{§1. Preamble}

As has been stressed recently by droves of authors, Heyting valued set theory could be conceptually interesting and technically useful to various areas of modern mathematics. Rousseau [10] has demonstrated that standard function theory of $n$ variables is no other than intuitionistic function theory of one variable over $\boldsymbol{C}^{n-1}$. This idea of internal-external transitions of the viewpoint has been prodded further by Nishimura [9], who showed that Sato hyperfunctions with $n$ holomorphic parameters are none other than those without parameters over $\mathbb{C}^{n}$. Takeuti and Titani [16] have pursued the same idea in the realm of complex manifolds to find out that vector bundles over a complex manifold are apartness vector spaces, that families of complex structures are simply complex manifolds, and so on. The present paper, belonging to this vein, aims at pursuing the idea in the arena of algebraic geometry. We show that fibred products of schemes over a base scheme are intuitionistically fibred products of schemes over an affine scheme, that projective spaces over a scheme are simply projective spaces over a ring, and that higher direct images of

Communicated by S. Takasu, October 30, 1986.

* Institute of Mathematics, University of Tsukuba, Ibaraki 305, Japan. 
sheaves are just cohomology groups with sheaf coefficients.

To this end, we need to reconstruct algebraic geometry from an intuitionistic standpoint. We use, as our starting point, the complete Heyting algebra of radical ideals, whose importance in the context of topoi was noticed first by Tierney [17]. After reviewing some rudiments of Heyting valued set theory in Section 2, we approach the first concepts of scheme theory in Section 3. Section 4 is devoted to projective spaces, while Section 5 deals with higher direct images of sheaves. We do not intend this paper to be exhaustive at all. On the contrary, we restrict our discussion to some rudimental aspects of algebraic geometry, partly because the exhaustive treatment is surely beyond our power and time, but mainly because we intend this paper to be illustratively interesting both to logicians who may not be versed in algebraic geometry and to algebraic geometricians who may not be experienced in Heyting valued set theory at all.

\section{$\S 2$. Heyting Valued Set Theory}

In this section we review some rudiments of Heyting valued set theory of Takeuti and Titani [14] together with some relevant materials of Fourman and Scott $[1]$.

\subsection{Intuitionistic Set Theory}

By $Z F_{I}$ we mean a first order intuitionistic theory with a unary relation symbol $\mathrm{E}$ and two binary relation symbols $\in$ and = satisfying the following nonlogical axioms:

(A1) Equality axioms : $u=u$,

$$
\begin{aligned}
& u=v \rightarrow v=u, \\
& u=v \wedge \varphi(u) \rightarrow \varphi(v), \text { and }(\mathrm{E} u \vee \mathrm{E} v \rightarrow u=v) \rightarrow u=\imath .
\end{aligned}
$$

(A2) Extensionality: $\dot{\forall} z(z \in u \leftrightarrow z \in v) \wedge(\mathrm{E} u \leftrightarrow \mathrm{E} v) \rightarrow u=v$.

(A3) Pairing: $\dot{\exists} z \dot{\forall} x(x \in z \leftrightarrow x=u \vee x=v)$.

(A4) Union: $\dot{\exists} v \dot{\forall} x(x \in v \leftrightarrow \dot{\exists} y \in u(x \in y))$.

(A5) Power sets: $\dot{\exists} v \dot{\forall} x(x \in v \leftrightarrow \dot{\forall} y \in u(y \in x))$.

(A6) $\varepsilon$-induction: $\dot{\forall} x(\dot{\forall} y \in x \varphi(y) \rightarrow \varphi(x)) \rightarrow \dot{\forall} x \varphi(x)$.

(A7) Infinity: $\dot{\exists} v(\dot{\exists} x \in v \wedge \dot{\forall} x \in v \dot{\exists} y \in v(x \in y))$.

(A8) Separation: $\dot{\exists} v \dot{\forall} x(x \in v \leftrightarrow x \in u \wedge \varphi(x))$.

(A9) Collection: $\dot{\exists} v(\dot{\forall} x \in u \dot{\exists} y \varphi(x, y) \rightarrow \dot{\forall} x \in u \dot{\exists} y \in v \varphi(x, y)$ ) 
In the above list $\dot{\forall} x \cdots$ and $\dot{\exists} x \cdots$ are abbreviations of $\forall x(\mathrm{E} x \rightarrow \cdots)$ and $\exists x(\mathrm{E} x \wedge \cdots)$. Since $\forall x$ and $\exists x$ will usually appear in these forms, we will often write $\forall x$ and $\exists x$ simply for $\dot{\forall} x$ and $\dot{\exists} x$.

\subsection{Heyting Valued Models}

Let $V$ be an arbitrary universe of $Z_{\mathrm{I}}$ and let $\Omega$ be a cHa (complete Heyting algebra) in $V$. For each ordinal $\alpha$ we define $V_{\alpha}^{(2)}$ inductively to be the set of all ordered pairs $\langle u, \mathrm{E} u\rangle$ such that:

(1) $\mathrm{E} u \in \Omega$;

(2) $u$ is an $\Omega$-valued function defined on a subset $\mathscr{D}(u)$ of $V_{\beta}^{(\Omega)}$ for some ordinal $\beta<\alpha$;

(3) $x \in \mathscr{D}(u)(u(x) \leqq \mathrm{E} u \wedge \mathrm{E} x)$.

Now $V^{(\Omega)}$ is defined to be the class $\bigcup_{a \in \mathrm{O}_{\mathrm{n}}} V_{a}^{(\Omega)}$, which is to be called an $\left(\Omega\right.$-valued) sheaf model, can be considered to be a Heyting valued model of $Z F_{\mathrm{I}}$ by defining $\llbracket \mathrm{E} u \rrbracket$ with

(1) $\llbracket \mathrm{E} u \rrbracket=\mathrm{E} u$,

and by defining $\llbracket u \in v \rrbracket$ and $\llbracket u=v \rrbracket$ with the following simultaneous induction

(2) $\llbracket u \in v \rrbracket=\bigvee_{y \in \mathscr{D}(v)}(v(y) \wedge \llbracket u=y \rrbracket)$,

(3) $\llbracket u=v \rrbracket=\wedge_{x \in \mathscr{D}(u)}(u(x) \rightarrow \llbracket x \in v \rrbracket) \wedge \wedge_{y \in \mathscr{D}(v)}(v(y) \rightarrow \llbracket y \in u \rrbracket) \wedge(\mathrm{E} u \leftrightarrow \mathrm{E} v)$,

and then by assigning a Heyting value $\llbracket \varphi \rrbracket$ to each nonatomic sentence $\varphi$ inductively as follows:

(4) $\llbracket \varphi_{1} \wedge \varphi_{2} \rrbracket=\llbracket \varphi_{1} \rrbracket \wedge \llbracket \varphi_{2} \rrbracket$,

(5) $\llbracket \varphi_{1} \vee \varphi_{2} \rrbracket=\llbracket \varphi_{1} \rrbracket \vee \llbracket \varphi_{2} \rrbracket$,

(6) $\llbracket \varphi_{1} \rightarrow \varphi_{2} \rrbracket=\llbracket \varphi_{1} \rrbracket \rightarrow \llbracket \varphi_{2} \rrbracket$,

(7) $\llbracket\rceil \varphi \rrbracket=\neg \llbracket \varphi \rrbracket$,

(8) $\llbracket \exists x\left(\varphi(x) \rrbracket=\bigvee_{x \in V(\Omega)} \llbracket \varphi(x) \rrbracket\right.$,

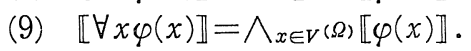

Now we have

Theorem 2.2.1. $V^{(\Omega)}$ is a model of $\mathrm{ZF}_{\mathrm{I}}$.

The class $V$ can be embedded into $V^{(\Omega)}$ by transfinite induction as follows: $\check{y}=\{\langle\check{x}, T\rangle \mid x \in y\}$ and $\mathrm{E} \check{y}=\top$ for $y \in V$, where $T$ is the empty join of $\Omega$.

For $u \in V^{(\Omega)}$ and $p \in \Omega$, we define $u\left\lceil p\right.$ to be the element of $V^{(\Omega)}$ such that 
and

$$
\begin{aligned}
& \mathscr{D}(u \Gamma p)=\{x \Gamma p \mid x \in \mathscr{D}(u)\} \\
& (u \Gamma p)(x \Gamma p)=\vee\{u(t) \wedge p \mid t \in \mathscr{D}(u), t\ulcorner p=x \Gamma p\} \quad \text { for } \quad x \in \mathscr{D}(u)
\end{aligned}
$$

$$
\mathrm{E}(u \Gamma p)=\mathrm{E} u \wedge p
$$

In the sequel we implicitly identify $x, y \in V^{(\Omega)}$ time and again provided $\llbracket x=y \rrbracket=T$.

\subsection{Sheaves over Complete Heyting Algebras}

A presheaf over a $\mathrm{cHa} \Omega$ is a triple $\langle\mathscr{T}, \mathrm{E}, \Gamma\rangle$ of a set $\mathscr{I}$ and two functions $\mathrm{E}: \mathscr{F} \rightarrow \Omega$ and $\Gamma: \mathscr{F} \times \Omega \rightarrow \mathscr{I}$ with the following properties:

(1) $a \Gamma \perp=b\lceil\perp$;

(2) $a \Gamma \mathrm{E} a=a$;

(3) $\mathrm{E}(a \Gamma p)=\mathrm{E} a \wedge p$;

(4) $(a\ulcorner p) \Gamma q=a \Gamma(p \wedge q)$.

For convenience we often say simply that $\mathscr{I}$ is a presheaf over $\Omega$ without mentioning $\mathrm{E}$ and $\Gamma$ explicitly. Members $a, b$ of a presheaf $\mathscr{I}$ over $\Omega$ are said to be compatible whenever $a\ulcorner\mathrm{E} b=b\lceil\mathrm{E} a$. A subset of $\mathscr{I}$ whose members are pairwise compatible is called compatible. A presheaf $\mathscr{I}$ over $\Omega$ is called a sheaf over $\Omega$ if for any compatible subset $D$ of $\mathscr{T}$ there exists a unique $c \in \mathscr{F}$ such that:

(1) $d \in D$ implies $c\lceil\mathrm{E} d=d$;

(2) $\mathrm{E} c=\vee\{\mathrm{E} d \mid d \in D\}$.

The subset $\{a \in \mathscr{F} \mid \mathrm{E} a=p\}$ is denoted by $\Gamma(p, \mathscr{I})$ for any $p \in \Omega$.

Given two sheaves $\mathscr{F}, \mathcal{G}$ over $\Omega$, a function $f$ from $\mathcal{G}$ to $\mathscr{g}$ is called a sheaf morphism provided:

(1) $\mathrm{E} a=\mathrm{E} f(a)$;

(2) $f(a)\lceil p=f(a \Gamma p)$.

The sheaf morphism $f$ restricted to $\Gamma(p, \mathscr{I})$ and whose range is considered to be $\Gamma(p, \mathcal{G})$ is denoted by $f_{p}$.

Given a cHa $\Omega$, a sheaf $\mathscr{F}$ over $\Omega$ and $p \in \Omega$, we write $\left.\Omega\right|_{p}$ for

and $\left.\mathscr{F}\right|_{p}$ for

$$
\{q \in \Omega \mid q \leqq p\}
$$

$$
\{a \in \mathscr{F} \mid \mathrm{E} a \leqq p\} .
$$

$\left.\Omega\right|_{p}$ inherits the cHa structure from $\Omega$ and $\left.\mathscr{I}\right|_{p}$ can be regarded as a sheaf over $\left.\Omega\right|_{p}$.

In this paper we consider empty and binary meets $(T$ and $\wedge$ ) and arbitrary 
joins $(V)$ as primitive in our definition of cHa. This means in particular that a cHa morphism is defined to be a function from one $\mathrm{cHa}$ to another preserving these primitive operations. Each cHa morphism $f^{*}: \Omega \rightarrow H$ has the right adjoint $f_{*}: H \rightarrow \Omega$ characterized by :

$$
f^{*}(p) \leqq q \leftrightarrow p \leqq f_{*}(q)
$$

for any $p \in \Omega$ and any $q \in H$. We use freely other standard notations and terminologies of sheaf theory, for which the reader is referred to Fourman and Scott [1] and other standard textbooks on sheaf theory. In particular, given a cHa morphism $f^{*}: \Omega \rightarrow H$, a sheaf $\mathscr{F}$ on $\Omega$ and a sheaf $\mathcal{G}$ on $H$ we can speak of the direct image sheaf $f_{*} \mathcal{G}$ on $\Omega$ and the inverse image sheaf $f^{*} \mathscr{F}$ on $H$, for which the reader is referred to Fourman and Scott $[1 ; 6.4$ and 9.4].

Given a cHa $\Omega$, elements of $V^{(\Omega)}$ and sheaves over $\Omega$ are essentially the same. Indeed we have

Theorem 2.3.1. For any $u \in V^{(\Omega)}, \hat{u}=\left\{x \in V^{(\Omega)} \mid \llbracket x \in u \rrbracket=\mathrm{E} x\right\}$ is a sheaf to be called the sheaf represented by $u$. Conversely, for any sheaf $\mathcal{I}$ over $\Omega$, there is an element $u \in V^{(\Omega)}$ such that the sheaf $\hat{u}$ represented by $u$ is isomorphic to $F$.

Similarly we have

Theorem 2.3.2. Let $u_{1}, u_{2} \subseteq V^{(\Omega)}$. Then any function $f: u_{1} \rightarrow u_{2}$ in $V^{(\Omega)}$ renders a unique function $\hat{f}: \hat{\iota}_{1} \rightarrow \hat{\imath}_{2}$ such that for each $a \in \hat{\iota}_{1}$,

$$
\mathrm{E} a=\mathrm{E} \hat{f}(a)=\llbracket\langle a, \hat{f}(a)\rangle \in f \rrbracket .
$$

This gives a bijective correspondence between functions $f: u_{1}-u_{2}$ in $V^{(\Omega)}$ and sheaf morphisms from $\hat{\imath}_{1}$ to $\hat{\imath}_{2}$.

\section{4. $\Omega$-Sets}

Let $\Omega$ be a cHa. An $\Omega$-s $s$ is a set $A$ equipped with an $\Omega$-relation $\llbracket \cdot \sim \cdot \mathbb{}: A \times A \rightarrow \Omega$ satisfying

(1) $\llbracket a \sim b \rrbracket=\llbracket b \sim a \rrbracket$, and

(2) $\llbracket a \sim b \rrbracket \wedge \llbracket b \sim c \rrbracket \leqq \llbracket a \sim c \rrbracket$.

Let $A$ be an $\Omega$-set. A singleton of $A^{-}$is a map $s: A \rightarrow \Omega$ such that

(1) $s(a) \wedge \llbracket a \sim b \rrbracket \leqq s(b)$, and

(2) $s(a) \wedge s(b) \leqq \llbracket a \sim b \rrbracket$

for all $a, b \in A$. For any $c \in A$, the map $a \rightarrow \llbracket a \sim c \rrbracket$ is a singleton, which is denoted by $\hat{c}_{\text {. }}$ The $\Omega$-set $A$ is cailed complete if every singleton occurs in this manner. An $\Omega$-set $A$ may not necessarily be complete, but the sheafification $\hat{A}$ of $A$ defined by 


$$
\hat{A}=\{s: A \rightarrow \Omega \mid s \text { is a singleton }\} \quad \text { with } \llbracket s \sim t \rrbracket=\vee\{s(a) \wedge t(a) \mid a \in A\}
$$

is always complete.

The relationship between sheaves over $\Omega$ and complete $\Omega$-sets is simple. Indeed we have

Theorem 2.4.1. Sheaves over $\Omega$ and complete $\Omega$-sets come to the same thing.

Thus we can speak of elements of $V^{(\Omega)}$, sheaves over $\Omega$ and complete $\Omega$ sets interchangeably. Exploiting this trinity, which of the three is most convenient will be preferably used on each occasion.

\section{$\S 3 . \quad$ Intuitionistic Algebraic Geometry I; First Concepts}

Throughout this and succeeding sections a ring always means a commutative ring with identity element 1 . And all homomorphisms of rings are supposed to take 1 to 1 . The discussion that follows (including the next two sections) is formalizable within the formal system $Z F_{\mathrm{I}}$.

A ringed $c H a$ is a pair $\left(\Omega, \Theta_{\Omega}\right)$ consisting of a $\mathrm{cHa} \Omega$ and a sheaf of rings $\mathcal{O}_{\Omega}$ on $\Omega$. A morphism of ringed cHas $f$ from $\left(H, \mathcal{O}_{H}\right)$ to $\left(\Omega, \mathcal{O}_{\Omega}\right)$ consists of a pair $\left(f^{*}, f^{\#}\right)$ of a cHa morphism $f^{*}$ from $\Omega$ to $H$ and a map $f^{\#}: \mathcal{O}_{\Omega} \rightarrow f_{*} \mathcal{O}_{H}$ of sheaves of rings on $\Omega$. The ringed $\mathrm{cHa}\left(\Omega, \Theta_{\Omega}\right)$ is called a locally ringed $\mathrm{cHa}$ if the sheaf $\mathcal{O}_{\Omega}$, regarded as a ring in $V^{(\Omega)}$, is a local ring. Given two locally ringed $\mathrm{cHas}\left(H, \mathcal{O}_{H}\right)$ and $\left(\Omega, \mathcal{O}_{\Omega}\right)$, a morphism of ringed cHas $\left(f^{*}, f^{\#}\right)$ from $\left(H, \mathcal{O}_{H}\right)$ to $\left(\Omega, \mathcal{O}_{\Omega}\right)$ is called a morphism of locally ringed cHas if $f^{\#}$, regarded as a homomorphism of rings in $V^{(\Omega)}$, is a local homomorphism of local rings.

Next we introduce a cHa version of affine schemes simply by gathering the scattered materials of Fourman and Scott [1]. Given a ring $A$, we denote by $\operatorname{Spec} A$ the cHa of all radical ideals of $A$, for which the reader is referred to Fourman and Scott $[1 ; 2.15$, pp. 327-328]. Let $\Omega=\operatorname{Spec} A$. Then the ring $A$ can be made an $\Omega$-set by defining $\llbracket a \sim b \rrbracket$ to be

$$
\left\{c \in A \mid c^{n} a=c^{n} b \text { for some } n>0\right\} .
$$

We also introduce a nonstandard relation of separation on the same set by defining $\llbracket a \neq b \rrbracket$ to be

$$
\left\{c \in A \mid c^{n} \in(a-b) A \text { for some } n>0\right\} .
$$

Using this $\Omega$-set $A$ with separation $\llbracket \cdot \neq \cdot \rrbracket$, we make the set

$$
S=\left\{a b^{-1} \mid a, b \in A\right\}
$$

an $\Omega$-set by defining $\llbracket a b^{-1} \sim c d^{-1} \rrbracket$ to be

$$
\llbracket b \neq 0 \wedge d \neq 0 \wedge \exists e \neq 0 \quad e(a d-b c) \sim 0 \rrbracket .
$$


Finally the completion of this $\Omega$-set renders the desired sheaf $\mathcal{O}_{\operatorname{Spec} A}$. The pair ( $\operatorname{Spec} A, O_{\operatorname{Spec} A}$ ), which is known to be locally ringed $\mathrm{cHa}$, is called the spectrum of $A$.

An affine scheme is a locally ringed $\mathrm{cHa}\left(\Omega, \mathcal{O}_{\Omega}\right)$ which is isomorphic (as a locally ringed $\mathrm{cHa}$ ) to the spectrum of some ring. A scheme is a locally ringed $\mathrm{cHa}\left(\Omega, \mathcal{O}_{\Omega}\right)$ for which there exists a family $\left\{p_{i}\right\}_{i \in I}$ of elements of $\Omega$ such that $\left(\left.\Omega\right|_{p_{i}},\left.\mathcal{O}_{\Omega}\right|_{p_{i}}\right)$ is an affine scheme for any $i \in I$ and $T=\bigvee_{i \in I} p_{i}$. We call $\Omega$ the underlying $\mathrm{cHa}$ of the scheme $\left(\Omega, \mathcal{O}_{\Omega}\right)$ and $\mathcal{O}_{\Omega}$ its structure sheaf. By abuse of notation we will often write simply $\Omega$ for the scheme $\left(\Omega, \Theta_{\Omega}\right)$. A morphism of schemes is a morphism as locally ringed cHas and similarly for isomorphisms.

Let $\Omega$ be a scheme. Then a scheme over $\Omega$ or simply an $\Omega$-scheme is a scheme $H$ together with a morphism from $H$ to $\Omega$. In this context the scheme $\Omega$ is called the base scheme of $(H, f)$ and the morphism $f$ is called the structure morphism of $(H, f)$. We will often say that $H$ is an $\Omega$-scheme without explicitly mentioning the structure morphism $f$. Given two $\Omega$-schemes $H_{1}$ and $H_{2}$ with their structure morphisms $f_{1}$ and $f_{2}$ respectively, an $\Omega$-morphism from $H_{1}$ to $H_{2}$ is a morphism of schemes $\varphi$ from $H_{1}$ to $H_{2}$ such that the following diagram is commutative.

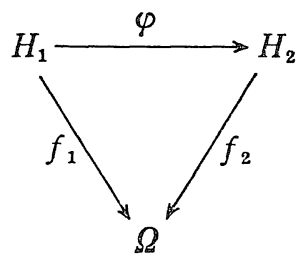

Now let $\varphi: A \rightarrow B$ be a ring homomorphism. Then we would like to define its associated morplisin of schemes ${ }^{a} \varphi=\left(f^{*}, f^{\#}\right)$ from ( $\operatorname{Spec} B, \mathcal{O}_{\operatorname{Spec} B}$ ) to ( $\operatorname{Spec} A$, $\left.\Theta_{\text {Spec } A}\right)$. First we define $f^{*}: \operatorname{Spec} A \rightarrow \operatorname{Spec} B$ to be

$$
f *(\mathfrak{p})=\sqrt{\varphi(\mathfrak{p}) B}
$$

for any $\mathfrak{p} \in \operatorname{Spec} A$. I. e., $f^{*}(\mathfrak{p})$ is the least radical ideal containing $\varphi(\mathfrak{p})$. Then we have

Proposition 3.1. $f^{*}$ is a cHa morphism.

Proof. Trivially $f^{*}\left(\bigvee_{i} \mathfrak{p}_{i}\right)=\bigvee_{i} f^{*}\left(\mathfrak{p}_{2}\right)$. Since $\varphi(1)=1, f^{*}(A)=B$. Thus it remains to show that $f^{*}(\mathfrak{p} \cap \mathfrak{q})=f^{*}(\mathfrak{p}) \cap f^{*}(\mathfrak{q})$. Obviously $f^{*}(\mathfrak{p} \cap \mathfrak{q}) \subset f^{*}(\mathfrak{p}) \cap f^{*}(\mathfrak{q})$. Now let $c \in f^{*}(\mathfrak{p}) \cap f^{*}(\mathfrak{q})$. Then

$$
\begin{aligned}
& c^{m}=x_{1} \varphi\left(a_{1}\right)+\cdots+x_{k} \varphi\left(a_{k}\right) \\
& c^{n}=y_{1} \varphi\left(b_{1}\right)+\cdots+y_{l} \varphi\left(b_{l}\right)
\end{aligned}
$$

for some positive integers $k, l, m, n,\left\{a_{1}, \cdots, a_{k}\right\} \subset \mathfrak{p},\left\{b_{1}, \cdots, b_{l}\right\} \subset q$, and 
$\left\{x_{1}, \cdots, x_{k}, y_{1}, \cdots, y_{l}\right\} \subset B$. Multiplying both sides, we have

$$
c^{m+n}=\sum_{\substack{1 \leq \imath \leq k \\ 1 \leqq j \leqq l}} x_{i} y_{\jmath} \varphi\left(a_{\imath} b_{j}\right) .
$$

Since the right-hand side apparently belongs to $f^{*}(p \cap q)$, the proof is complete.

Now it remains to define $f^{\#}$. Since

$$
\begin{aligned}
f^{*}(\llbracket a \neq 0 \rrbracket) & =f^{*}(\sqrt{a A}) \\
& =\sqrt{\varphi(a) B} \\
& =\llbracket \varphi(a) \neq 0 \rrbracket
\end{aligned}
$$

for any $a \subseteq A$, the correspondence

$$
a b^{-1} \mapsto \varphi(a) \varphi(b)^{-1}
$$

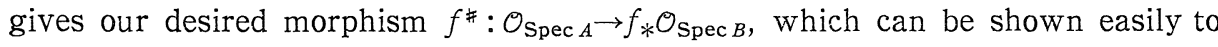
be a local homomorphism in $V^{(\operatorname{Spec} A)}$. Thus we have the desired morphism of schemes ${ }^{a} \varphi=\left(f^{*}, f^{\#}\right)$, for which we have

Theorem 3.2. Let $A$ and $B$ be rings. Then the correspondence $\varphi^{a} \varphi$ gives a bijection between $\operatorname{Hom}(A, B)$ and $\operatorname{Hom}(\operatorname{Spec} B, \operatorname{Spec} A)$, where $\operatorname{Hom}(A, B)$ denotes the totality of ring homomorphisms from $A$ to $B$ and $\operatorname{Hom}(\operatorname{Spec} B, \operatorname{Spec} A$ ) denotes the totality of morphisms of schemes from $\operatorname{Spec} B$ to $\operatorname{Spec} A$.

Proof. Given an arbitrary ring homomorphism $\varphi: A \rightarrow B$, we can recover $\varphi$ from ${ }^{a} \varphi=\left(f^{*}, f^{\#}\right)$ as $f_{A}^{\#}$, since $\Gamma\left(A, \mathcal{O}_{\operatorname{Spec} A}\right)=A$ and $\Gamma\left(B, \mathcal{O}_{\operatorname{Spec} B}\right)=B$. Therefore the correspondence $\varphi^{\mapsto}{ }^{a} \varphi$ is injective. Now suppose that an arbitrary morphism of $\operatorname{schemes}\left(f^{*}, f^{\#}\right)$ from $\left(\operatorname{Spec} B, \mathcal{O}_{\operatorname{Spec} B}\right)$ to $\left(\operatorname{Spec} A, \mathcal{O}_{\operatorname{Spec} A}\right)$ is given. Let $\varphi=f_{A}^{\#}$. Since $f^{\#}$, regarded as a ring homomorphism in $V^{(\operatorname{Spec} A)}$, is a local homomorphism of local rings, we have

$$
\begin{aligned}
f^{*}(\sqrt{a A}) & =f *(\llbracket a \neq 0 \rrbracket) \\
& =f *(\llbracket \varphi(a) \neq 0 \rrbracket) \\
& =\sqrt{\varphi(a) B}
\end{aligned}
$$

for any $a \in A$. Therefore we can see easily that $\left(f^{*}, f^{*}\right)={ }^{a} \varphi$.

This result can be generalized readily to

Theorem 3.3. Let $A$ be a ring and let $\left(\Omega, O_{\Omega}\right)$ be a scheme. Then, by assigning $f_{A}^{*}$ to each morphism of schemes $\left(f^{*}, f^{*}\right)$ from $\left(\Omega, \Theta_{\Omega}\right)$ to $\left(\operatorname{Spec} A, \Theta_{\operatorname{Spec} A}\right)$, we obtain a bijection between $\operatorname{Hom}(\Omega, \operatorname{Spec} A)$ and $\operatorname{Hom}\left(A, \Gamma\left(T, \mathcal{O}_{\Omega}\right)\right)$, where $\operatorname{Hom}(\Omega, \operatorname{Spec} A)$ is the totality of morphisms of schemes from $\left(\Omega, \mathcal{O}_{\Omega}\right)$ to $(\operatorname{Spec} A$, 
$\left.\mathcal{O}_{\mathrm{Spec} A}\right)$ and $\operatorname{Hom}\left(A, \Gamma\left(T, \mathcal{O}_{\Omega}\right)\right)$ is the totality of ring homomorphisms from $A$ to $\Gamma\left(\mathrm{T}, \Omega_{\Omega}\right)$.

\section{Proof. Essentially the same as in Iitaka [4; Theorem 1.15].}

Let $f^{*}: \Omega \rightarrow H$ be a cHa morphism. Then we can embed the $\mathrm{cHa} H$ and many constructs on $H$ into $V^{(\Omega)}$. Here we prefer using sheaf representations, which can be regarded as elements of $V^{(\Omega)}$, as was explained in the previous section. First of all, we define the sheaf $\tilde{H}$ to be

$$
\begin{aligned}
& \left\{(q, p) \in H \times \Omega \mid q \leqq f^{*}(p)\right\}, \quad \text { where } \\
& \mathrm{E}(q, p)=p \text { and } \\
& (q, p)\left\ulcorner p^{\prime}=\left(q \wedge f^{*}\left(p^{\prime}\right), p \wedge p^{\prime}\right) .\right.
\end{aligned}
$$

It is easy to see that $\widetilde{H}$ is indeed a $\mathrm{cHa}$ in $V^{(\Omega)}$.

Next, given a sheaf $\mathscr{I}$ on $H$, we define $\tilde{I}$ to be

$$
\begin{aligned}
& \{(a, q, p) \in \mathscr{I} \times H \times \Omega \mid \mathrm{E} a=q \text { and }(q, p) \in \widetilde{H}\}, \quad \text { where } \\
& \mathrm{E}(a, q, p)=p \text { and } \\
& (a, q, p) \Gamma p^{\prime}=\left(a \Gamma f^{*}\left(p^{\prime}\right), q \wedge f^{*}\left(p^{\prime}\right), p \wedge p^{\prime}\right) .
\end{aligned}
$$

It is easy to see that $\tilde{\Psi}$ is a sheaf on $\tilde{H}$ in $V^{(\Omega)}$. Given two sheaves $\mathscr{F}_{1}, \mathscr{F}_{2}$ over $H$ and a sheaf morphism $\omega$ from $\mathscr{I}_{1}$ to $\mathscr{I}_{2}$, we define $\widetilde{\omega}$ to be

$$
\widetilde{\omega}(a, q, p)=(\omega(a), q, p)
$$

for any $(a, q, p) \in \tilde{\mathscr{T}}_{1}$. $\widetilde{\omega}$ can be considered a sheaf morphism from $\tilde{\mathscr{T}}_{1}$ to $\tilde{\mathscr{I}}_{2}$ in $V^{(\Omega)}$.

Now suppose that we are given three cHa morphisms $f_{1}^{*}: \Omega \rightarrow H_{1}, f_{2}^{*}: \Omega \rightarrow H_{2}$, and $\varphi^{*}: H_{1} \rightarrow H_{2}$ such that the following diagram is commutative.

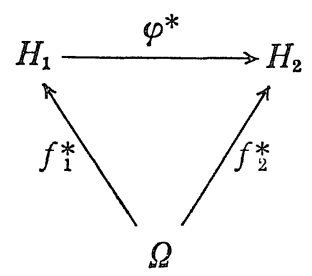

Then $\tilde{\varphi}^{*}$, defined to be

$$
\tilde{\varphi}^{*}(q, p)=\left(\varphi^{*}(q), p\right)
$$

for any $(q, p) \in \widetilde{H}_{1}$, can be shown easily to be a cHa morphism in $V^{(\Omega)}$. The right adjoint of $\tilde{\varphi}^{*}$ is denoted somewhat ambiguously by $\tilde{\varphi}_{*}$. This ambiguity does not cause confusion at all, since $\left(\varphi_{*}\right)^{\sim}$ is essentially the same as $\tilde{\varphi}_{*}$.

One of the most interesting applications of these constructs is in 
Theorem 3.4. Let $\left(\Omega, \mathcal{O}_{\Omega}\right)$ be a scheme. Since the identity morphism of schemes $\left(\operatorname{id}_{\Omega}, \operatorname{id}_{\mathcal{O}_{\Omega}}\right):\left(\Omega, \mathcal{O}_{\Omega}\right) \rightarrow\left(\Omega, \mathcal{O}_{\Omega}\right)$ subsumes the identity cHa morphism $\operatorname{id}_{\Omega}: \Omega \rightarrow$ $\Omega$ we can apply the above constructions to $\left(\Omega, \Theta_{\Omega}\right)$ to obtain a ringed $c H a\left(\tilde{\Omega}, \widetilde{O}_{\Omega}\right)$ in $V^{(\Omega)}$. Then we can assert that this ringed $c H a\left(\widetilde{\Omega}, \widetilde{O}_{\Omega}\right)$ is even an affine scheme in $V^{(\Omega)}$.

Proof. We will show that the sheaf $\mathcal{O}_{\Omega}$, regarded as a ring in $V^{(\Omega)}$, gives rise to the desired affine scheme $\left(\Omega, \mathcal{O}_{\Omega}\right)$, assuming without loss of generality that the given scheme $\left(\Omega, \mathcal{O}_{\Omega}\right)$ is an affine scheme, say, ( $\operatorname{Spec} A, \mathcal{O}_{\operatorname{Spec} A}$ ) for some ring $A$. Since $\{\sqrt{a A} \mid a \in A\}$ is a base for $\Omega$, it suffices to show that for each $a \in A$,

(I) $\Gamma\left(\sqrt{a A},(\operatorname{Spec} A)^{\sim}\right)=\Gamma\left(\sqrt{a A}, \operatorname{Spec} \Theta_{\operatorname{Spec} A}\right)$, and

(II) $\Gamma\left(\sqrt{a A},\left(\mathcal{O}_{\mathrm{Spec} A}\right)^{2}\right)=\Gamma\left(\sqrt{a A}, \mathcal{O}_{\mathrm{Spec}} \mathcal{O}_{\operatorname{Spec} A}\right)$,

where in the right-hand sides of (I) and (II),

(1) $\mathcal{O}_{\mathrm{Spec} A}$ is regarded as a ring in $V^{(\Omega)}$,

(2) the ring $\mathcal{O}_{\mathrm{Spec} A}$ gives rise to a ringed $\mathrm{cHa}\left(\operatorname{Spec} \mathcal{O}_{\mathrm{Spec} A}, \mathcal{O}_{\mathrm{Spec}} \Theta_{\operatorname{Sepc} A}\right)$ in $V^{(\Omega)}$, and

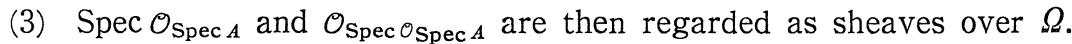

Here we deal only with ( I), leaving (II) to the reader. By definition,

$$
\Gamma\left(\sqrt{a A},(\operatorname{Spec} A)^{\sim}\right)=\{p \in \operatorname{Spec} A \mid p \subset \sqrt{a A}\} .
$$

According to Fourman and Scott [1; Theorem 6.12],

$$
\Gamma\left(\sqrt{a A}, \mathcal{O}_{\operatorname{Spec} A}\right)=A_{a},
$$

where $A_{a}$ is the localization of $A$ with respect to the multiplicative system $\{1\} \cup\left\{a^{n} \mid n>0\right\}$. Therefore (I) follows from the following analogue of litaka [4; Proposition 1.2 (iv)].

(III) The cHa $\operatorname{Spec} A_{a}$ is isomorphic to $\{\mathfrak{p} \in \operatorname{Spec} A \mid \mathfrak{p} \subset \sqrt{a A}\}$.

To see (III), it is sufficient to note that the correspondence

$$
\mathfrak{q} \mapsto\left\{x a^{-m} \mid x \in \mathfrak{q}, m>0\right\}
$$

from $\{\mathfrak{p} \in \operatorname{Spec} A \mid \mathfrak{p} \subset \sqrt{a A}\}$ to $\operatorname{Spec} A_{a}$ is bijective.

This result can be generalized to

Theorem 3.5. Let $\left(H, \mathcal{O}_{H}\right)$ be a scheme over a base scheme $\left(\Omega, \mathcal{O}_{\Omega}\right)$ with $\left(f^{*}, f^{\#}\right)$ as its structure morphism. Then the ringed $c H a\left(\tilde{H}, \widetilde{O}_{H}\right)$ is a scheme in $V^{(\Omega)}$.

Proof. We can assume without loss of generality that the schemes $\left(H, \mathcal{O}_{H}\right)$ 
and $\left(\Omega, \mathcal{O}_{\Omega}\right)$ are affine schemes, say, $\left(\operatorname{Spec} B, \mathcal{O}_{\mathrm{Spec} B}\right)$ and $\left(\operatorname{Spec} A, \mathcal{O}_{\mathrm{Spec} A}\right)$ respectively for some rings $A, B$. Then, by dint of Theorem 3.2 , we can assume also that the structure morphism $\left(f^{*}, f^{\#}\right)$ derives from a ring homomorphism $\varphi: A \rightarrow B$ as ${ }^{a} \varphi$. Under these assumptions we will show that the scheme $\left(\widetilde{H}, \widetilde{O}_{H}\right)$ is an affine scheme in $V^{(\Omega)}$ by demonstrating that the sheaf $f_{*} \mathcal{O}_{\operatorname{Spec} B}$ over $\Omega$, if regarded as a ring in $V^{(\Omega)}$, gives rise to the scheme $\left(\tilde{H} . \widetilde{\mathcal{O}}_{H}\right)$. Since

$$
\begin{aligned}
\Gamma\left(\sqrt{a A}, f_{*} \Theta_{\mathrm{Spec} B}\right) & =\Gamma\left(\sqrt{\varphi(a) B}, \Theta_{\mathrm{Spec} B}\right) \\
& =B_{\varphi(a)}
\end{aligned}
$$

for each $a \in A$, we can establish by the same method of Theorem 3.4 that

(I) $\Gamma\left(\sqrt{a A},(\operatorname{Spec} B)^{\sim}\right)=\Gamma\left(\sqrt{a A}, \operatorname{Spec}\left(f_{*} \mathcal{O}_{\operatorname{Spec} B}\right)\right)$, and

(II) $\Gamma\left(\sqrt{a A},\left(\mathcal{O}_{\mathrm{Spec} B}\right)^{\sim}\right)=\Gamma\left(\sqrt{a A}, \mathcal{O}_{\mathrm{Spec}\left(f_{*}, O_{\operatorname{Spec} B}\right)}\right)$.

As $\{\sqrt{a A} \mid a \in A\}$ is a base for the $\mathrm{cHa} \operatorname{Spec} A$, the proof is complete.

Now given a scheme $\left(\Omega, \mathcal{O}_{\Omega}\right)$ as a base scheme, two $\Omega$-schemes $\left(H_{1}, \mathcal{O}_{H_{1}}\right)$, $\left(H_{2}, \mathcal{O}_{H_{2}}\right)$ with their structure morphisms $f_{1}=\left(f_{1}^{*}, f_{1}^{\#}\right), f_{2}=\left(f_{2}^{*}, f_{2}^{*}\right)$, and an $\Omega$ morphism $\varphi=\left(\varphi^{*}, \varphi^{\#}\right)$ from $\left(H_{1}, \mathcal{O}_{H_{1}}\right)$ to $\left(H_{2}, \mathcal{O}_{H_{2}}\right)$, we can embed the three morphisms $f_{1}, f_{2}$ and $\varphi$ into $V^{(\Omega)}$ as $\tilde{f}_{1}=\left(\tilde{f}_{1}^{*}, \tilde{f}_{1}^{\#}\right), \tilde{f}_{2}=\left(\tilde{f}_{2}^{*}, \tilde{f}_{3}^{\#}\right)$ and $\tilde{\varphi}=\left(\tilde{\varphi}^{*}, \tilde{\varphi}^{\#}\right)$ respectively, and we have the following commutative diagram:

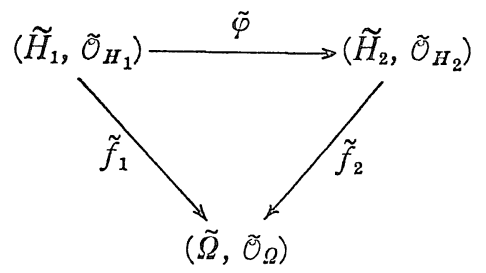

We summarize these considerations in

Theorem 3.6. $\tilde{\varphi}$ is an $\tilde{\Omega}$-morphism in $V^{(\Omega)}$.

To conclude this section, we consider the notion of the fibred product of two schemes $H_{1}$ and $H_{2}$ over the same base scheme $\Omega$, which is to be denoted by $H_{1} \times{ }_{\Omega} H_{2}$. For this notion we have

Theorem 3.7. Given an arbitray scheme $\Omega$ (as the base scineme) and two $\Omega$ schemes $H_{1}$ and $H_{2}$, the fibred product $H_{1} \times{ }_{\Omega} H_{2}$ always cxis.s.

Proof. Essentially the same as in Iitaka [4; Theorem 1.16]. In other words the standard proof is intuitionistically valid.

The following result is of considerable interest, though its proof is ele- 
mentary.

Theorem 3.8. Given a scheme $\Omega$ (as the base scheme) and two $\Omega$-schemes $H_{1}$ and $H_{2}$, we have

$$
\left(H_{1} \times{ }_{\Omega} H_{2}\right)^{\sim}=\widetilde{H}_{1} \times \widetilde{\Omega} \widetilde{H}_{2}
$$

in $V^{(\Omega)}$.

Proof. Follows readily from the definitions.

\section{§4. Intuitionistic Algebraic Geometry II; Projective Spaces}

Let $A$ be a ring and let $S$ be the polynomial ring $A\left[x_{0}, \cdots, x_{n}\right]$ over $A$ in $n+1$ indeterminates $x_{0}, \cdots, x_{n}$. We denote by $S_{+}$the ideal generated by $n_{0}, \cdots, x_{n}$, which is apparently a homogeneous radical ideal. We denote by Proj $S$ the set of all the homogeneous radical ideals of $S$ that are contained in $S_{+}$. Then we have the following.

Theorem 4.1. Proj $S$ is a cHa.

Proof. Similar to that of Fourman and Scott $[1 ; 2.15$, pp. 327-328] claiming that $\operatorname{Spec} A$ is a $\mathrm{cHa}$. The details are left to the reader.

Next we would like to define the structure sheaf $\mathcal{O}_{\operatorname{Proj} S}$ on Proj $S$. To this end, some definitions are in order. Let $\Omega=\operatorname{Proj} S$. For each natural number $m$, let $S_{m}$ be the set of all the homogeneous polynomials of degree $m$. The set $S_{m}$ can be made an $\Omega$-set by defining $\llbracket f \sim g \rrbracket$ to be the ideal

$$
\left\{h \in S_{+} \mid h^{k} f=h^{k} g \text { for some } k>0\right\},
$$

which is apparently a homogeneous radical ideal. We next introduce a nonstandard relation of separation $\neq$ on $S_{m}$ by defining $\llbracket f \neq g \rrbracket$ to be

$$
\left\{h \in S_{+} \mid h^{k} \in(f-g) S \text { for some } k>0\right\} \text {. }
$$

Let $T$ be the set of $f g^{-1}$ 's for all pairs of homogeneous polynomials $f, g$ of the same degree. This set can be made an $\Omega$-set by defining $\llbracket f_{1} g_{1}^{-1} \sim f_{2} g_{2}^{-1} \rrbracket$ to be

$$
\llbracket g_{1} \neq 0 \wedge g_{2} \neq 0 \wedge \exists \text { homogeneous } h \neq 0 h\left(f_{2} g_{1}-f_{1} g_{2}\right) \sim 0 \rrbracket .
$$

Now the structure sheaf $\mathcal{O}_{\text {Pro: } S}$ shall be the sheaf obtainable from the $\Omega$-set $T$. Then we have

Theorem 4.2. For any homogeneous polynomial $f \in S_{+}$,

$$
\Gamma\left(\sqrt{f S}, \mathcal{O}_{\operatorname{Proj} S}\right)=S_{(f)} .
$$


where $\sqrt{f S}$ is the least homogeneous radical ideal containing $f$ and

$S_{(f)}=\left\{g h^{-1} \in S_{f} \mid g\right.$ and $h$ are homogeneous polynomials of tho same degree $\}$

with $S_{f}$ being the localizatin of $S$ with respect to the multiplicative system $\{1\} \cup$ $\left\{f^{m} \mid m>0\right\}$.

Proof. Similar to that of Fourman and Scott [1; Theorem 6.12, p. 377].

In order to show that $\left(\operatorname{Proj} S, \mathcal{O}_{\operatorname{Proj} S}\right)$ is a scheme, it remains to demonstrate that $\left(\operatorname{Proj} S, \mathcal{O}_{\operatorname{Proj} S}\right)$ is covered with affine schemes, which follows from

Theorem 4.3. Let $R$ be the polynomial ring $A\left[x_{1}, \cdots, x_{n}\right]$ over $A$ in $n$ indeterminates $x_{1}, \cdots, x_{n}$. Then $\left(\operatorname{Spec} R, \mathcal{O}_{\operatorname{Spec} R}\right)$ and $\left(\left.\operatorname{Proj} S\right|_{\sqrt{x_{0} S},} \mathcal{O}_{\operatorname{Proj} S \mid \sqrt{x_{0} S}}\right)$ are isomorphic as ringed cHas.

Proof. First we will show that $\operatorname{Spec} R$ and Proj $S \mid \sqrt{x_{0} S}$ are isomorphic cHas. Using the notation of Zariski and Samuel [18; p. 179], we denote by ${ }^{h} f$ the homogenized polynomial of any $f \in R$. I. e.,

$$
{ }^{h} f\left(x_{0}, \cdots, x_{n}\right)=x_{0}^{\partial(f)} f\left(x_{1} x_{0}^{-1}, \cdots, x_{n} x_{0}^{-1}\right),
$$

where $\partial(f)$ denotes the degree of $f$. Slightly different from Zariski and Samuel $\left[18 ;\right.$ p. 180], when we are given an ideal $\mathfrak{a}$ of $R$, we denote by ${ }^{h} \mathfrak{a}$ the homogeneous ideal generated by the forms $x_{0}^{m} \cdot{ }^{h} f(m \geqq 1, f \in \mathfrak{a})$. It is easy to see that whenever $\mathfrak{a}$ is a radical ideal, then ${ }^{h} \mathfrak{a}$ is a homogeneous radical ideal, which follows from the simple observation that $f^{m} \in \mathfrak{a}$ implies $f \in \mathfrak{a}$ for any homogeneous polynomial $f \in S$. To realize that the mapping $\mathfrak{a} \mapsto{ }^{h} \mathfrak{a}$ gives an isomorphism between Spec $R$ and $\left.\operatorname{Proj}\right|_{\sqrt{x_{0} S}}$, it suffices to note that $x_{\cup}^{m} \cdot f \in \mathfrak{G}(m \geqq 1)$ implies $x_{0} f \in \mathfrak{b}$ for any $\left.\mathfrak{b} \in \operatorname{Proj} S\right|_{\sqrt{x_{0} S}}$, since $\left(x_{0} f\right)^{m}=\left(x_{0}^{m} f\right) \cdot f^{m-1}$.

Now it remains to show that the isomorphism between cHas $\operatorname{Spec} R$ and Proj $\left.S\right|_{\sqrt{x_{0} S}}$ can be extended to an isomorphism between ringed cHas (Spec $R$, $\mathcal{O}_{\text {Spec } R}$ ) and (Proj $\left.S\right|_{\sqrt{x_{0} S}},\left.\mathcal{O}_{\operatorname{Proj} S}\right|_{\sqrt{x_{0} S}}$ ), since the family $\{\sqrt{f R} \mid f \in R\}$ is a base

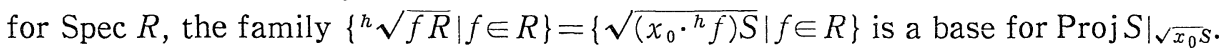
Since $R_{f}$ and $S_{\left(x_{0}, h_{f}\right)}$ are naturally isomorphic, the proof is complete in view of Theorem 4.2 of this paper and Theorem 6.12 of Fourman and Scott [1].

Corollary 4.4. The ringed $\mathrm{cHa}$ (Proj $\left.S, \mathcal{O}_{\operatorname{Proj} S}\right)$ is a scizme, which is to be deinoted by $\boldsymbol{P}_{A}^{n}$ and to be called the projective $n$-space over $A$.

Since any scheme $\Omega$ is naturally considered a scheme over $\operatorname{Spec} \mathbb{Z}$ and $\mathbb{P}_{Z}^{n}$ is also considered a scheme over $\operatorname{Spec} Z$, the projective n-sface over the scheme $\Omega$ can be defined as $\Omega \times{ }_{\operatorname{Spec} Z} P_{Z}^{n}$ and denoted by $\mathbb{P}_{\Omega}^{n}$. Then we have

Theorem 4.5. Let $\left(\Omega, \Theta_{\Omega}\right)$ be an arbitrary scheme. Then $P_{\Omega}^{n}$ is a scheme 
over $\Omega$ and we have

$$
\left(\boldsymbol{P}_{\Omega}^{n}\right)^{\sim}=\boldsymbol{P}_{\odot \Omega}^{n}
$$

in $V^{(\Omega)}$, where $\mathcal{O}_{\Omega}$ in the right-hand side is regarded as a ring in $V^{(\Omega)}$.

Proof. Follows from the definitions. The reader is also referred to the readable exposition of Hartshorne [3; pp. 160-169] for this and further research.

\section{§5. Intuitionistic Algebraic Geometry III; Higher Direct Images of Sheaves}

Given a cHa $\Omega$ and a sheaf $\mathscr{F}$ of abelian groups on $\Omega$, cohomology groups $H^{n}(\Omega, \mathscr{I})$ are defined by imitating the usual construction of Čech cohomology with sheaf coefficients, for which the reader is referred to Takeuti and Titani $[16 ; 2.3]$.

Let $f^{*}: \Omega \rightarrow H$ be a cHa morphism and $\mathscr{T}$ be a sheaf of abelian groups on $H$. Then the higher direct images $R^{n} f_{*}(\mathscr{I})(n \geqq 0)$ are defined to be the sheaves associated to the presheaves

$$
p \mapsto H^{n}\left(\left.H\right|_{f^{*}(p)},\left.\mathscr{F}\right|_{f^{*}(p)}\right)
$$

on $\Omega$.

By the way, as we have discussed in Section 3, the cHa $H$ and the sheaf $\mathscr{I}$ on $H$ can be embedded into $V^{(\Omega)}$ as $\tilde{H}$ and $\tilde{\mathscr{I}}$ respectively. Then we have

Theorem 5.1. The cohomology groups $H^{n}(\tilde{H}, \tilde{\Psi})$ in $V^{(\Omega)}$ are externally the higher direct images $R^{n} f_{*}(\mathscr{I})(n \geqq 0)$.

Proof. Follows readily from the definition of $R^{n} f_{*}$ and the construction of $\widetilde{H}$ and $\tilde{\mathscr{T}}$

\section{$\S 6$. Concluding Remarks}

As we have seen so far, our algebraic geometry differs from the standard one in several critical points. The most important difference is that the ambient logic is not classical but intuitionistic. Since prime ideals are not well-behaved creatures in intuitionistic reasoning, this differece affects greatly our choice of building blocks of scheme theory. Indeed we were forced to define affine schemes by using radical ideals in place of prime ideals, which renders the second distinctive feature of our scheme theory. The third distinctive feature of our scheme theory is that topological spaces and their related constructs should be replaced by corresponding cHas and their related constructs. In particular, socalled Zariski topology plays no role in this new context.

The best companion of algebraic geometry has been commutative algebra, 
which is expected to be the case in our intuitionistic context. Therefore full development of intuitionistic algebraic geometry should be accompanied by some corresponding maturity of intuitionistic commutative algebra, which seems to be in an embryonic stage at present. The birth of intuitionistic algebraic geometry will presumably accelerate the development of intuitionistic algebra.

Last but not least, Grothendieck has stressed in EGA [Eléments de Géometrie Algébrique] the tenet that the main object of algebraic geometry is not schemes but morphisms of schemes. As we have seen, our Heyting valued approach to algebraic geometry is completely in resonance with his relativistic philosophy.

\section{References}

[1] Fourman, M.P. and Scott, D.S., Sheaves and logic, Lecture Notes in Math., 753 (1979), 302-401, Springer, Berlin.

[2] Hakim, M., Topos annelés et schémas relatifs, Springer, Berlin, 1972.

[3] Hartshorne, R., Algebraic geometry, Springer, New York, 1977.

[4] Iitaka, S., Algebraic geometry, Springer, New York, 1982.

[5] Johnstone, P.T., Topos theory, Academic Press, London, 1977.

[6] - Rings, fields, and spectra, Journal of Algebra, 49 (1977), 238-260.

[7] Lawvere, F.W., Continuously variable sets; algebraic geometry=geometric logic, in Logic Colloquim '73, ed. by Rose, H.E. and Shepherdson, J.C., North-Holland, Amsterdam, 1975, pp. 135-156.

[8] Nagata, M., Theory of commutative algebras (in Japanese), Kinokuniya, Tokyo, 1974.

[9] Nishimura, H., Heyting valued set theory and Sato hyperfunctions, Publ. RIMS, Kyoto Univ., 22 (1986), 801-811.

[10] Rousseau, C., Topos theory and complex analysis, Lecture Notes in Math., 753 (1979), 623-659, Springer, Berlin.

[11] Ruitenburg, W.B.G., Intuitionistic algebra, theory and sheaf models, dissertation.

[12] Scott, D. S., Identity and existence in intuitionistic logic, Lecture Notes in Math., 753 (1979), 660-696, Springer, Berlin.

[13] Takeuti, G., Intuitionistic set theory (in Japanese), Kinokuniya, Tokyo, 1980.

[14] Takeuti, G. and Titani, S., Heyting valued universes of intuitionistic set theory, Lecture Notes in Math., 891 (1980), Springer, Berlin, pp. 189-306.

[15] — Globalization of intuitionistic set theory, preprint.

[16] - Global intuitionistic analysis, preprint.

[17] Tierney, M., On the spectrum of a ringed topos, in Algebra, Topology and Category Theory: a collection of papers in honor of Samuel Eilenberg, ed. by Heller, A. and Tierney, M, Academic Press, New York, 1976, pp. 189-210.

[18] Zariski, O. and Samuel, P., Commutative algebra, Vol. II, Van Nostrand, Princeton, N. J., 1960. 
